\title{
Lateralization of the Vertebrate Brain: Taking the Side of Model Systems
}

\author{
Marnie E. Halpern, ${ }^{1 \star}$ Onur Güntürkün, ${ }^{2 \star}$ William D. Hopkins, ${ }^{3,4}$ and Lesley J. Rogers ${ }^{5}$ \\ ${ }^{1}$ Carnegie Institution of Washington, Department of Embryology, Baltimore, Maryland 21218, ${ }^{2}$ Department of Biopsychology, Institute for Cognitive \\ Neuroscience, Faculty of Psychology, Ruhr-Universität Bochum, 44780 Bochum, Germany, ${ }^{3}$ Berry College, Mount Berry, Georgia 30149, ${ }^{4}$ Division of \\ Psychobiology, Yerkes National Primate Research Center, Emory University, Atlanta, Georgia 30322, and ${ }^{5}$ Centre for Neuroscience and Animal Behaviour, \\ University of New England, School of Biological, Biomedical, and Molecular Sciences, Armidale, New South Wales 2351, Australia
}

Popular culture, from movies, advertising, to self-help books, is captivated by left-brain/right-brain differences and how these might influence our personalities, moods, and capabilities. Considering the interest in understanding the scientific basis for lateralized neural functions, it is surprising that model systems have not played a more dominant role in research on brain asymmetry. The long-held view that laterality is unique to the human cortex has been supplanted by overwhelming evidence of left-right ( $\mathrm{L}-\mathrm{R})$ differences in neuroanatomy and neural processing across vertebrate and even some invertebrate species. Recent inroads into the genetic, anatomical, and behavioral specializations of the brains of model research animals have refocused attention on the evolution and advantage of brain laterality. Technical advances in arenas as diverse as molecular biology and brain imaging have sparked the identification of localized sites of asymmetry. Developmental studies have probed the influence of experience on the generation of lateralization. The goal of this review is to highlight some of the multipronged approaches and diverse models currently being used to explore how the left brain functions differently from the right.

\section{Genetic approaches: the zebrafish model}

How anatomical asymmetry is imposed on a seemingly bilaterally symmetric structure, the vertebrate neural tube, is unknown. Fundamental studies on the origin of body axes, and the molecular pathways that confer positional information and polarity onto a developing embryo, have laid the groundwork for deciphering what makes the left side of the nervous system different from the right. In vertebrates, selective expression of the TGF $\beta$ family member Nodal on the left side of the early embryo mediates the asymmetrical morphogenesis and placement of the visceral organs through activation of a signaling cascade that has not yet been fully resolved (Schier, 2003; Levin, 2005). The discovery that Nodal signaling is also transiently activated on the left side of

\footnotetext{
Received Aug. 15, 2005; accepted Aug. 17, 2005.

Some of the described studies were supported by National Institutes of Health Grants HD042215 (M.E.H.) and NS036605 and NS042867 (W.D.H.), Deutsche Forschungsgemeinschaft Sonderforschungsbereich 509 (0.G.), and a Discovery Grant from the Australian Research Council (L.J.R.). We thank Josh Gamse for his expertise on laterality in films.

${ }^{*} M . E . H$. and $0 . G$. contributed equally to this review.

Correspondence should be addressed to Marnie E. Halpern, Department of Embryology, 3520 San Martin Drive, Carnegie Institution of Washington, Baltimore, MD 21218. E-mail: halpern@ciwemb.edu.

DOI:10.1523/JNEUROSCI.3439-05.2005

Copyright $\odot 2005$ Society for Neuroscience $\quad$ 0270-6474/05/2510351-07\$15.00/0
}

the brain of zebrafish embryos (Rebagliati et al., 1998; Sampath et al., 1998) opened the way to molecular genetic studies on the development and regulation of brain asymmetry.

Expression of cyclops, one of three zebrafish Nodal-related genes, and of other components of the signaling pathway, is restricted to the left half of the presumptive pineal organ in the developing dorsal diencephalon (Concha et al., 2000, Liang et al. 2000). Processes important to maintain visceral asymmetry, or mutations that perturb them, influence asymmetric gene expression in the diencephalon, suggesting some commonality in L-R axis formation throughout the developing embryo (Bisgrove et al., 2000). Loss of Nodal pathway activation leads to a randomization in the $\mathrm{L}-\mathrm{R}$ position of the adult pineal organ and of the parapineal, an accessory organ that typically (>95\%) develops to the left of the pineal in embryonic zebrafish (Concha et al., 2000; Liang et al., 2000; Gamse et al., 2002). Subsequent studies have demonstrated that the parapineal affects the gene expression profile and morphology of the adjacent brain nucleus, the left habenula (Concha et al., 2003; Gamse et al., 2003, 2005). Thus, directional asymmetry of one relatively inconspicuous structure, the parapineal, has consequences on the differentiation of a neighboring brain region and has even farther-reaching effects on neuronal projections and connectivity.

The habenular nuclei of zebrafish (equivalent to the dorsal habenulae of amphibians and medial habenulae of mammals) reside within an evolutionarily conserved conduction system that relays telencephalic input to the midbrain (Sutherland, 1982). Habenular efferents course ventrocaudally through the fasciculus retroflexus to innervate the midbrain target, the interpeduncular nucleus (IPN) (Fig. 1A,B). Surprisingly, the left and right habenular neurons of zebrafish project along different dorsoventral extents of the target (Aizawa et al., 2005; Gamse et al., 2005), and, when laterality is lost in the epithalamic region (e.g., after specific ablation of the parapineal), the dorsoventral pattern of habenular connectivity is also perturbed (Gamse et al., 2005). Disruption of asymmetry in the diencephalon, therefore, affects stereotypic input to the midbrain target and, presumably, output to hindbrain nuclei and other regions that normally elicit behavioral responses.

Although the habenulointerpeduncular projection has been well characterized anatomically in many vertebrates, its functional role is less well defined. Hence, understanding why laterality has been superimposed on this conduction system at the 
individual and populational levels will require a greater appreciation of the behaviors under its influence. Mutational screens in zebrafish to uncover the genes that control differentiation of the epithalamus could provide useful tools to explore function, although mutants will need to have regionally restricted deficits and survive long enough for comprehensive behavioral assays. With gene disruption methods, it is also possible to reverse the direction of epithalamic asymmetry in the zebrafish, which should allow neuroanatomical biases to be correlated with lateralized behaviors. A recent study with a strain of zebrafish that shows a higher than normal frequency of $\mathrm{L}-\mathrm{R}$ reversals in visceral and epithalamic asymmetry suggests that larvae also exhibit a change in some but not all directional visual behaviors (Barth et al., 2005).

Another puzzle is that, although the habenular region of many amphibians, reptiles, and birds displays pronounced biochemical and morphological asymmetries (Concha and Wilson, 2001), left and right habenular efferents seem to innervate the target similarly. Rather than projecting along different dorsoventral extents of the IPN as in teleosts, left and right axons traverse the IPN in a mirror image pattern in the brains of juvenile frogs and salamanders (Y.-S. Kuan and M. E. Halpern, unpublished results). This finding indicates that laterality of the epithalamic region does not necessarily translate into differential innervation of the target in all species or that L-R differences in the habenular nuclei might provoke subtler changes elsewhere in the brain. Clearly, additional comparative analyses are required to resolve the extent of conservation and purpose of asymmetry in the habenulointerpeduncular conduction system.

Although progress has been rapid and genetic strategies are powerful, it remains to be demonstrated whether the zebrafish diencephalon is the most appropriate model for determining the basis of laterality in the developing mammalian cortex. Asymmetric expression of components of the Nodal signaling pathway has not been discovered in the brains of any other vertebrate species so far, suggesting that other molecular mechanisms could be responsible. Screens of random genes for their spatial transcriptional patterns during zebrafish embryonic and larval development have netted a number that show differential L-R expression in the epithalamus (Gamse et al., 2003, 2005) (C. Thisse, B. Thisse, and M. E. Halpern, unpublished observations). More direct, large-scale approaches to detect molecular asymmetry in the vertebrate brain have proven challenging because L-R differences in gene expression can be very transient as well as confined to limited brain regions and difficult to distinguish. However, a recent study using a sensitive molecular biology method [SAGE (serial analysis of gene expression)] uncovered 27 differentially expressed genes in the human cortex, one of which was also found
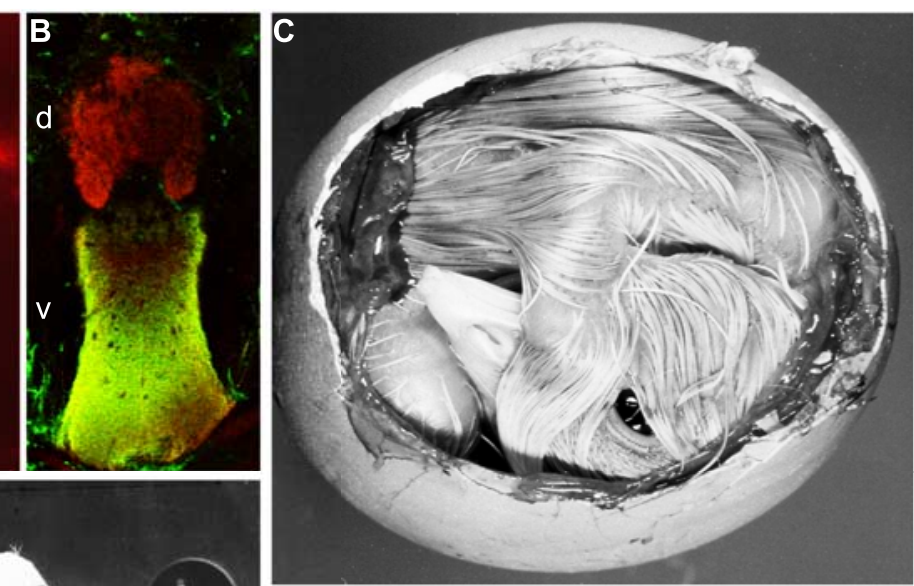

Figure 1. Left-right asymmetry in vertebrate nervous systems. A, Asymmetric protein distribution in the dorsal habenular (hed) project along the entire dorsoventral extent of the IPN, whereas right habenular axon green) project only ventrally. Shown are transverse sections of an adult brain immunolabeled for two proteins that are expressed (as in $\boldsymbol{A}$ ) or right habenular neurons and their axons (image courtesy of Y.-S. Kuan). C, Head position of a day 18 chick embryo within the egg results in lateralized light input and asymmetry in the developing visual system (Rogers, 1990). Only the right eye is exposed to light in both chick and pigeon (data not shown) embryos. $\boldsymbol{D}$, Adult pigeon with cap over the right eye in front of two pecking keys showing patterns. To the bottom are some of the other 725 abstract patterns that the animals 1990). $\boldsymbol{E}$, MRI images depicting L-R asymmetry in the motor-hand area of the precentral gyrus of different primates. All brains are shown to scale and oriented as for the human.

to be expressed asymmetrically in the developing mouse brain (Sun et al., 2005). Comparative analyses across vertebrate species will serve as an effective way of validating gene expression asymmetries and testing their significance in neural development.

\section{Visual system asymmetry: avian models}

The ontogeny of lateralization has been investigated in two avian species, the precocial domestic chick (Gallus gallus) and the altricial pigeon (Columba livia). In both species, development of visual object discrimination asymmetry is triggered by exposure of the embryo to light (chick, Rogers and Sink, 1988; pigeon, Skiba et al., 2002). The later-stage embryo adopts a turned posture, consistently in the same direction, so that the left eye is occluded by its own body and the right eye remains exposed to light that enters through the egg shell and air sac membranes (Fig. 1C).

In both species, asymmetric light stimulation leads to the development of visual pathway asymmetry and lateralized visual behavior. Accordingly, incubation of eggs in darkness prevents the development of visual lateralization (chick, Rogers, 1990, 1997; Rogers and Bolden, 1991; pigeon, Skiba et al., 2002). In fact, 
the direction of lateralization can be reversed by withdrawing the head of a late-stage chick embryo from the egg, applying a patch to the right eye, and stimulating the left eye by light. Normally, a chick exposed to light before hatching is able to find food grains scattered on a background of small pebbles when it uses its right eye but less so, or not at all, with the left eye. The reverse is the case when only the embryo's left eye has been exposed to light (Rogers, 1990). In the precocial chick, the sensitive period for reversal of lateralization by right eye occlusion extends only to the first day or two after hatching (Rogers, 1990). In altricial pigeons, the visual system is modifiable for a longer period after hatching. Therefore, occluding the right eye after hatching for $10 \mathrm{~d}$ can reverse functional asymmetry such that, as adults, the birds have superior object discrimination capabilities with the left eye (Manns and Güntürkün, 1999).

The developmental stage of the visual system at the time of asymmetric light stimulation is critical and likely explains observed differences between the two avian species. Only the visual pathway undergoing differentiation at the time of light exposure becomes asymmetrical. In the chicken, this is the thalamofugal visual system, equivalent to the geniculocortical system of mammals. Asymmetry is manifested as an increase in forebrain projections from the left side of the thalamus (fed by the lightstimulated right eye) compared with the right side (Rogers and Deng, 1999; Koshiba et al., 2003). Whereas the tectofugal system (equivalent to the mammalian colliculo-pulvino-extrastriate system) has mostly completed its development during the final prehatching days in chicks, it is immature in altricial pigeons during the comparable period and thus develops asymmetry as a result of asymmetrical embryonic light input. Left-right differences within the pigeons' tectofugal system occur at various levels, in different neurochemically defined cell populations (Manns and Güntürkün, 2003) and in the quantities of recrossing fibers in ascending projections (Güntürkün et al., 1998). Additionally, tectofugal bottom-up projections and descending top-down systems from the forebrain interact in a lateralized way in pigeons (Folta et al., 2004). In chicks (Rogers, 1990) and pigeons (Manns and Güntürkün, 1999), experimentally induced shifts in anatomical asymmetry are accompanied by changes in behavioral asymmetry.

Steroid hormone levels in the embryo are also important in the ontogeny of lateralization. Injection of estrogen (Rogers and Rajendra, 1993), testosterone (Schwarz and Rogers, 1992), or corticosterone (Rogers, 2005) into the chick egg prevents the development of asymmetry in response to light. The interaction between hormone levels and light exposure is the likely cause of sex differences in the degree of asymmetry [male chicks have greater asymmetry than females (Rajendra and Rogers, 1993)] and suggests an intriguing role for stress, by altering corticosterone levels, in modulating the strength of asymmetry. The latter may result from stress of the hen, thereby affecting the amount of corticosterone she deposits in the egg yolk (Schwabl, 1999), or by stress of the late-stage embryo, modulating its endogenous release of corticosterone (Deng and Rogers, 2005). Hence, prehatching events, occurring during a brief sensitive period, influence phenotypes that are likely to enhance survival in different environments.

Added to these interactive events during embryonic development, the rotation of the embryo during the hatching process itself affects lateralization of motor behavior. The turned embryo rotates counterclockwise, driven by the musculature on the right side of its body, as it cracks open the shell, a behavior that is an essential precursor of limb and turning biases after hatching ( Ca- sey and Martino, 2000). Hence, the late-stage chick embryo relies on body posture, visual stimulation, and its own motor activity during hatching to express lateralization after hatching. Disruption of any one of these critical contributions to the canalizing of development precludes the expression of a broad range of motor and visual lateralizations.

Once visual asymmetry is induced before hatching, it modulates various visual functions. In pigeons, visuocognitive processes are changed asymmetrically for the entire lifetime of the animal. A detailed analysis of pigeons learning to categorize hundreds of pictures with everyday scenes into those that contain a human figure or not shows that left and right hemispheres use complementary strategies (Vauclair et al., 2005a). The left hemisphere concentrates on local features and is able to generate categorical distinctions based on the invariant properties of the target stimulus. The right hemisphere, however, relies on the configuration and possibly the global cues of the stimuli and seems to base its decisions on a memory-based exemplar strategy (Fig. 1D). These cognitive dichotomies of the cerebral hemispheres are mostly shared by humans, suggesting that lateralized cognitive systems already defined the neural architecture of the common ancestor of birds and mammals (Laeng et al., 2003; Lux et al., 2004).

\section{Handedness and brain imaging: the primate model}

Humans are predominantly right-handed, and most righthanded individuals are left hemisphere dominant for language. This association has led many to argue that language dominance and handedness coevolved and are unique to hominid evolution. Convergent evidence from behavioral and neuroimaging studies strongly challenged this historical view and have caused many to reconsider the influence of biological and social factors on the evolution of asymmetry in the primate CNS.

Recent work has focused on laterality, primarily in chimpanzees, but data also have been collected from other nonhuman primate species, including gorillas, orangutans, bonobos, and some monkeys, primarily rhesus macaques. Among many traits, primates, and particularly chimpanzees, differ from mammals in that they have hands that are used in manipulative functions that incorporate an opposable thumb. Moreover, chimpanzees are well known for their tool-using abilities and complex social and communicative behaviors that make them an ideal species for comparative studies of behavioral and brain asymmetries. With respect to hand preferences, recent studies in a host of nonhuman primate species have revealed evidence of population level handedness (Ward and Hopkins, 1993). For example, during tasks requiring controlled posture and reaching, the majority of prosimians show left-handedness (Ward et al., 1993). In Old and New World monkeys as well as great apes, assessment of hand preferences for coordinated bimanual tasks has revealed consistent evidence of population level handedness (Hopkins, 2005; Vauclair et al., 2005b). Two interesting trends emerge. First, not all measures elicit population level handedness, suggesting that hand preferences are task specific. Second, in most studies, the proportion of right-to-left individuals is $\sim 2: 1$, a value that is much lower than the typical 8:1 or 9:1 ratio reported in human populations. Whether these differences reflect genetic or socio-cultural factors remains unresolved.

Behavioral asymmetries associated with the perception and production of communicative signals also have been documented in primates. Early work on monkeys demonstrated rightear advantages in the processing of species-specific vocalizations (Petersen et al., 1978), and more recent studies in macaques using 
a larger sample size have reported right-sided orienting asymmetries in response to different classes of acoustic stimuli (Hauser and Andersson, 1994; Hauser et al., 1998). Unilateral damage to the left but not the right hemisphere from experimental lesions can have a transient effect on auditory discrimination of pure tones (Dewson, 1977) and species-specific calls in macaques (Heffner and Heffner, 1984). A recent positron emission tomography study in macaques also reported a left-hemisphere asymmetry in the anterior temporal lobe in discrimination of speciesspecific calls (Poremba et al., 2004).

Fewer studies have examined asymmetries in the production of vocal signals than in their perception. Orofacial asymmetries during the production of species-specific vocalizations have been examined in marmosets, rhesus monkeys, and chimpanzees, and, in all three species, a right-hemisphere bias was reported. In contrast to the directionality of orofacial asymmetries, preferential use of the right-hand for manual gestures was described for chimpanzees (Hopkins et al., 2005). Moreover, preferential use of the right-hand for gesturing is enhanced when the manual actions are accompanied by a vocalization compared with when no vocalization is produced (Hopkins and Cantero, 2003; Hopkins et al., 2005). These data pertain to recent theories suggesting that the lateralized system for language and speech found in modern humans evolved from a lateralized manual communication system already present in the common ancestor of chimpanzees and early hominids (Corballis, 2002).

With the advent of modern imaging technologies, particularly magnetic resonance imaging (MRI), evidence of population level neuroanatomical asymmetries is becoming increasingly evident in nonhuman primates (Fig. $1 E$ ). In chimpanzees, lefthemisphere asymmetries have been reported in the language homologs of the brain, including the planum temporale and inferior frontal gyrus (Gannon et al., 1998; Cantalupo and Hopkins, 2001; Cantalupo et al., 2003). Great apes also show the classic rightfrontal, left-occipital petalia asymmetry (Pilcher et al., 2001), as well as right-hemisphere asymmetry in the hippocampus (Freeman et al., 2004). Both apes and monkeys have longer left Sylvian fissures (Yeni-Komshian and Benson, 1976; Hopkins et al., 2000).

A central goal in the primate studies has been to establish whether there are any neuroanatomical correlates of behavioral asymmetries. To date, most studies have primarily focused on the relationship between neuroanatomical asymmetries and handedness (Hopkins and Cantalupo, 2004). Two main findings have been revealed. First, unlike most findings reported in human subjects, handedness for noncommunicative behaviors is not associated with asymmetries in the language homologs of chimpanzees, including the planum temporale and inferior frontal gyrus; however, asymmetries in hand use for manual gesture do correlate with the inferior frontal gyrus. Right-handed gesturing chimpanzees have a larger left inferior frontal gyrus compared with non-right-handed gesturing chimpanzees. Second, hand preferences for noncommunicative behaviors do correlate with asymmetries in the dorsal portion of the precentral gyrus, referred to as the "knob," in which neural representation of the hand is located in the primary motor cortex in both chimpanzees and capuchin monkeys (Phillips and Sherwood, 2005). Thus, different behavioral measures of hand use correlate with asymmetries from distinct regions of the frontal lobe. These findings raise a number of questions regarding the evolutionary assumptions of the association between right-handedness and left-hemisphere dominance for language in early hominids. Another important implication is that potential differences in structure and function of the left and right cerebral hemispheres should be considered in research aimed at understanding the neural systems involved in motor, perceptual, and cognitive processes in animals.

\section{Evolutionary implications: the advantage of laterality}

Given the widespread occurrence of lateralization in vertebrates (Rogers and Andrew, 2002) and reports of lateralization in some invertebrate species (nematode, Hobert et al., 2002; octopus, Byrne et al., 2004; fruit fly, Pascual et al., 2004), it seems reasonable to assume that this characteristic must bestow a favorable selective advantage. Recent research has focused on discovering what this advantage might be. Here it is important to consider two kinds of lateralization separately because they may have different advantages or disadvantages: (1) lateralization of individuals without an overall bias for the individuals to be lateralized in the same direction, and (2) lateralization of individuals with the majority having the same direction of lateralization and hence generating a population bias.

Lateralization present in individuals without a directional bias in the population seems to enhance neural capacity, as postulated by researchers of lateralization in humans some years ago (Levy, 1977; Dunaif-Hattis, 1984). Recent experiments comparing the performance of lateralized chicks (exposed to light before hatching; see above) and chicks without lateralization of visual pathways or visual behavior (incubated in the dark) have lent support to this hypothesis (Rogers et al., 2004). The chicks were asked to perform two tasks simultaneously. One task, searching for food grains against a background of pebbles, engaged the right eye and left hemisphere (Deng and Rogers, 1997), and the other, being vigilant for a model predator, engaged the left eye and right hemisphere (Evans et al., 1993; Rogers, 2000). The lateralized chicks were able to find the food grains and also respond to a model predator moved over the cage (repeated presentations of the predator until the chick had pecked 60 times). This contrasted markedly to the performance of the nonlateralized chicks, which were unable to avoid pecking pebbles and also often missed detecting and responding to the overhead predator (Rogers et al., 2004). In fact, the nonlateralized chicks were so disturbed by the dual task that their performance deteriorated as the task progressed. Without the presentation of the predator, the nonlateralized chicks had no difficulty in finding the grain and not pecking at pebbles. Hence, their confusion arises only when they have to engage each hemisphere on a separate task. Monocular testing of lateralized and nonlateralized chicks on the same task has revealed that the superior ability of the lateralized chicks is attributable to the specific effect of light stimulation before hatching on the pathways fed by the right eye. In other words, light exposure of the right eye before hatching (see above) triggers developmental events that will put the right eye and left hemisphere in charge of foraging and the left eye and right hemisphere in charge of predator detection and/or recognition (Dharmaretnam and Rogers, 2005). These results strongly suggest that lateralization enhances neural capacity by allowing parallel and separate processing in the hemispheres. Indeed, light incubation in pigeons has been shown to induce different and hemisphere-specific behavioral (Skiba et al., 2002) and anatomical (Manns and Güntürkün, 2003) changes that result in functional specialization of each half brain.

Recent experiments (Rogers, 2005) have shown a similar result for common marmosets tested on a dual task of foraging and predator detection. Marmosets with weaker hand preferences, measured by their ability to pick up and hold food to the mouth, were slower to react to a model predator (hawk or snake) than 
were marmosets with stronger hand preferences, regardless of the direction of the lateralization. More strongly lateralized pigeons can find grain among pebbles more effectively than weakly lateralized ones (Güntürkün et al., 2000), and chimpanzees using one hand consistently to probe for termites are more efficient in gaining a catch than chimpanzees that alternate hands (McGrew and Marchant, 1999). Moreover, Pascual et al. (2004) found that fruit flies with an asymmetrical structure in the brain are better able to form a memory of a particular event, and recent work in fish demonstrates that lateralized individuals have a greater ability at spatial reorientation (Sovrano et al., 2005). It might seem, therefore, that left-right differentiation of structure and function enhances the capacity of a brain across a wide range of species, regardless of species variation in neural capacity.

This leaves us with a need to explain why lateralization with a directional bias in the population is so common among vertebrates, especially because some clear disadvantages spring to mind: for example, being more responsive to predators approaching on the left side and to prey on the right side (Rogers, 2002). Predators might exploit this bias at the population level, and so might prey. These side biases would disadvantage an individual and they would disadvantage a population even more so, because lateralized individuals might gain by grouping provided that they are not lateralized with a population bias. Vallortigara and Rogers (2005) have hypothesized that populational asymmetry might depend on social behavior; that is, social stability may result when individuals are lateralized in the same direction, thereby increasing the predictability of individual lateralized behavior (Rogers, 1990; Robins et al.,1998).

So far, two sets of empirical data support the hypothesis that populational asymmetry is correlated with social cohesion in gregarious animals. First, a study of 16 species of fish by Bisazza et al. (2000) found that only those species that shoal show population level lateralization for turning at a barrier, whereas nonshoaling species have individual but not population lateralization. Second, groups of lateralized chicks form more stable social hierarchies than groups of nonlateralized chicks (Rogers and Workman, 1989).

\section{Future direction of laterality research}

Cerebral asymmetries were long seen as a turning point in human evolution. Their occurrence was assumed to have reduced cognitive redundancy and so produced the cerebral power that defines us. Handedness, lateralization of speech, and other left-right differences therefore had to be uniquely human, accounting for a general reluctance to accept animal models. Now that diverse animal models have been well established, research on brain laterality is accelerating and may soon provide answers for several deep questions.

Although knowledge on the genetic mechanisms that contribute to symmetry breaking in the embryo has accumulated, the principles of how $\mathrm{L}-\mathrm{R}$ differences arise in the developing nervous system are far less understood. Zebrafish genetic studies provide an important first step to link lateralized gene activation with the development of left-right differences in neuroanatomy. Because asymmetries of the habenular system are widespread among vertebrates, so might be the identified lateralized genetic mechanisms. The challenge will be to determine whether a few widely conserved genetic networks organize the template for cerebral asymmetries in higher vertebrates.

Research on birds stresses the importance of the interplay of genetic and epigenetic mechanisms for the emergence of the adult asymmetry pattern. Because environmental information can modify, abolish, or even reverse the lateralized avian visual system, nongenetic factors seem to be far more powerful in shaping left-right differences than previously assumed. Human handedness might be one example in which nongenetic factors could substantially increase a less pronounced genetic bias to the right side. It is likely that the search for epigenetic mechanisms that modify cerebral asymmetries will soon complement present endeavors to identify lateralized genes.

Studies in chimpanzees are especially suited to test long-held assumptions about the uniqueness of human asymmetries. Experiments are already able to show that handedness and lateralized speech are most likely not causally linked but are part of a general brain asymmetry pattern common to most vertebrates. This could imply that few basic dichotomies of computation are at the core of cerebral specialization. Asymmetry of the human brain, including that for speech, could then simply be the human version of an ancient inherited pattern.

The causation of asymmetries remains the most difficult question: why are we lateralized? As outlined above, individuals seem to benefit from lateralization, and a common pattern of asymmetries within a population might foster social cohesion. At this level, in which behavioral neuroscience converges with evolutionary biology, laterality research is gaining momentum; however, only animal models have brought us so far so fast.

\section{References}

Aizawa H, Bianco IH, Hamaoka T, Miyashita T, Uemura O, Concha ML, Russell C, Wilson SW, Okamoto H (2005) A novel feature of CNS circuitry by which left-right information from the forebrain is laterotopically represented along the dorso-ventral axis of a midbrain target nucleus. Curr Biol 15:238-243.

Barth KA, Miklosi A, Watkins J, Bianco IH, Wilson SW, Andrew RJ (2005) fsi zebrafish show concordant reversal of laterality of viscera, neuroanatomy, and a subset of behavioral responses. Curr Biol 15:844-850.

Bisazza A, Cantalupo C, Capocchiano M, Vallortigara G (2000) Population lateralization and social behavior: a study with sixteen species of fish. Laterality 5:269-284.

Bisgrove BW, Essner JJ, Yost HJ (2000) Multiple pathways in the midline regulate concordant brain, heart and gut left-right asymmetry. Development 127:3567-3579.

Byrne RA, Kuba MJ, Meisel DV (2004) Lateralized eye use in Octopus vulgaris shows antisymmetrical distribution. Animal Behav 68:1107-1114.

Cantalupo C, Hopkins WD (2001) Asymmetric Broca's area in great apes. Nature 414:505.

Cantalupo C, Pilcher D, Hopkins WD (2003) Are asymmetries in sylvian fissure length associated with the planum temporale? Neuropsychologia 41:1975-1981.

Casey MB, Martino C (2000) Asymmetrical hatching behaviours influence the development of postnatal laterality in domestic chicks (Gallus gallus). Dev Psychobiol 34:1-12.

Concha ML, Wilson SW (2001) Asymmetry in the epithalamus of vertebrates. J Anat 199:63-84.

Concha ML, Burdine RD, Russell C, Schier AF, Wilson SW (2000) A nodal signaling pathway regulates the laterality of neuroanatomical asymmetries in the zebrafish forebrain. Neuron 28:399-409.

Concha ML, Russell C, Regan JC, Tawk M, Sidi S, Gilmour DT, Kapsimali M, Sumoy L, Goldstone K, Amaya E, Kimelman D, Nicolson T, Grunder S, Gomperts M, Clarke JD, Wilson SW (2003) Local tissue interactions across the dorsal midline of the forebrain establish CNS laterality. Neuron 39:423-438.

Corballis MC (2002) From hand to mouth: the origins of language. Princeton: Princeton University.

Deng C, Rogers LJ (1997) Differential contributions of the two visual pathways to functional lateralization in chicks. Behav Brain Res 87:173-182.

Deng C, Rogers LJ (2005) Corticosterone treatment of the chick embryo affects light-stimulated development of the thalamofugal visual pathway. Behav Brain Res 159:63-71.

Dewson JH (1977) Preliminary evidence of hemispheric asymmetry of auditory function in monkeys. In: Lateralization in the nervous system 
(Harnad S, Doty RW, Goldstein L, Jaynes J, Krauthamer G, eds), pp 63-74. New York: Academic.

Dharmaretnam M, Rogers LJ (2005) Hemispheric specialization and dual processing in strongly versus weakly lateralized chicks. Behav Brain Res 162:62-70.

Dunaif-Hattis J (1984) Doubling the brain. New York: Lang.

Evans CS, Evans L, Marler P (1993) On the meaning of alarm calls: functional references in an avian vocal system. Anim Behav 46:23-28.

Folta K, Diekamp B, Güntürkün O (2004) Asymmetrical modes of visual bottom-up and top-down integration in the thalamic nucleus rotundus of pigeons. J Neurosci 24:9475-9485.

Freeman H, Cantalupo C, Hopkins WD (2004) Asymmetries in the hippocampus and amygdala of chimpanzees (Pan troglodytes). Behav Neurosci 118:1460-1465.

Gamse JT, Shen YC, Thisse C, Thisse B, Raymond PA, Halpern ME, Liang JO (2002) Otx5 regulates genes that show circadian expression in the zebrafish pineal complex. Nat Genet 30:117-121.

Gamse JT, Thisse C, Thisse B, Halpern ME (2003) The parapineal mediates left-right asymmetry in the zebrafish diencephalon. Development 130:1059-1068.

Gamse JT, Kuan YS, Macurak M, Thisse C, Thisse B, Brösamle C, Halpern ME (2005) Directional asymmetry of the zebrafish epithalamus guides dorsoventral innervation of the midbrain target. Development, 132:4869-4881.

Gannon PJ, Holloway RL, Broadfield DC, Braun AR (1998) Asymmetry of chimpanzee planum temporale: Humanlike pattern of Wernicke's brain language area homologue. Science 279:220-222.

Güntürkün O, Hellmann B, Melsbach G, Prior H (1998) Asymmetries of representation in the visual system of pigeons. NeuroReport 9:4127-4130.

Güntürkün O, Diekamp B, Manns M, Nottelmann F, Prior H, Schwarz A, Skiba M (2000) Asymmetry pays: visual lateralization improves discrimination success in pigeons. Curr Biol 10:1079-1081.

Hauser MD, Andersson K (1994) Left hemisphere dominance for processing vocalizations in adult, but not infant, rhesus monkeys: field experiments. Proc Natl Acad Sci USA 91:3946-3948.

Hauser MD, Agnetta B, Perez C (1998) Orienting asymmetries in rhesus monkeys: the effect of time-domain changes on acoustic perception. Anim Behav 56:41-47.

Heffner HE, Heffner RS (1984) Temporal lobe lesions and perception of species-specific vocalizations by macaques. Science 226:75-76.

Hobert O, Johnston Jr RJ, Chang S (2002) Left-right asymmetry in the nervous system: the Caenorhabditis elegans model. Nat Rev Neurosci 3:629-640

Hopkins WD (2005) Chimpanzee right-handedness: internal and external validity in the assessment of hand use. Cortex, in press.

Hopkins WD, Cantalupo C (2004) Handedness in chimpanzees is associated with asymmetries in the primary motor but not with homologous language areas. Behav Neurosci 118:1176-1183.

Hopkins WD, Cantero M (2003) The influence of vocalizations on preferential hand use in gestural communication by chimpanzees. Dev Sci 6:55-61.

Hopkins WD, Pilcher DL, MacGregor L (2000) Sylvian fissure length asymmetries in primates revisited: a comparative MRI study. Brain Behav Evol 56:293-299.

Hopkins WD, Russell J, Freeman H, Buehler N, Reynolds E, Schapiro SJ (2005) The distribution and development of handedness for manual gestures in captive chimpanzees (Pan troglodytes). Psychol Sci 16:487-493.

Koshiba M, Nakamura S, Deng C, Rogers LJ (2003) Light-dependent development of asymmetry in the ipsilateral and contralateral thalamofugal visual projections of the chick. Neurosci Lett 336:81-84.

Laeng B, Zarrinpar A, Kosslyn SM (2003) Do separate processes identify objects as exemplars versus members of basic-level categories? Evidence from hemispheric specialization. Brain Cogn 53:15-27.

Levin M (2005) Left-right asymmetry in embryonic development: a comprehensive review. Mech Dev 122:3-25.

Levy J (1977) The mammalian brain and the adaptive advantage of cerebral asymmetry. Ann NY Acad Sci 299:264-272.

Liang JO, Etheridge A, Hantsoo L, Rubinstein AL, Nowak SJ, Izpisua Belmonte JC, Halpern ME (2000) Asymmetric nodal signaling in the zebrafish diencephalon positions the pineal organ. Development 127:5101-5112.

Lux S, Marshall JC, Ritzl A, Weiss PH, Pietrzyk U, Shah NJ, Zilles K, Fink GR (2004) A functional magnetic resonance imaging study of local/global processing with stimulus presentation in the peripheral visual hemifields. Neuroscience 124:113-120.

Manns M, Güntürkün O (1999) Monocular deprivation alters the direction of functional and morphological asymmetries in the pigeon's visual system. Behav Neurosci 113:1-10.

Manns M, Güntürkün O (2003) Light experience induces differential asymmetry patterns of GABA- and parvalbumine-positive cells in the pigeon's visual midbrain. J Chem Neuroanat 25:249-259.

McGrew WC, Marchant LF (1999) Laterality of hand use pays off in foraging success for wild chimpanzees. Primates 40:509-513.

Pascual A, Huang K-L, Nevue J, Préat T (2004) Brain asymmetry and longterm memory. Nature 427:605-606.

Petersen MR, Beecher MD, Zoloth SR, Moody DB, Stebbins WC (1978) Neural lateralization of species-specific vocalizations in Japanese macaques (Macaca fuscata). Science 202:324-327.

Phillips K, Sherwood CS (2005) Primary motor cortex asymmetry correlates with handedness in capuchin monkeys (Cebus apella). Behav Neurosci, in press.

Pilcher DL, Hammock EA, Hopkins WD (2001) Cerebral volumetric asymmetries in non-human primates: a magnetic resonance imaging study. Laterality 6:165-179.

Poremba A, Malloy M, Saunders RC, Carson RE, Herscovitch P, Mishkin M (2004) Species-specific calls evoke asymmetric activity in the monkey's temporal poles. Nature 427:448-451.

Rajendra S, Rogers LJ (1993) Asymmetry is present in the thalamofugal visual projections of female chicks. Exp Brain Res 92:542-544.

Rebagliati MR, Toyama R, Fricke C, Haffter P, Dawid IB (1998) Zebrafish nodal-related genes are implicated in axial patterning and establishing left-right asymmetry. Dev Biol 199:261-272.

Robins A, Lipollis G, Bisazza A, Vallortigara G, Rogers LJ (1998) Lateralization of agonistic responses and hind-limb use in toads. Anim Behav $56: 875-881$.

Rogers LJ (1990) Light input and the reversal of functional lateralization in the chicken brain. Behav Brain Res 38:211-221.

Rogers LJ (1997) Early experiential effects on laterality: research on chicks has relevance to other species. Laterality 2:199-219.

Rogers LJ (2000) Evolution of hemispheric specialisation: advantages and disadvantages. Brain Lang 73:236-253.

Rogers LJ (2002) Lateralization in vertebrates: its early evolution, general pattern and development. In: Advances in the study of behavior 31 (Slater PJB, Rosenblatt J, Snowdon C, Roper TJ, eds), pp 107-162. San Diego: Academic.

Rogers LJ (2005) Cognitive and social advantages of having a lateralized brain. In: Behavioral and morphological asymmetries in vertebrates (Malashichev Y, Deckel AW, eds). Georgetown, Washington, DC: Landes Bioscience, in press.

Rogers LJ, Andrew RJ (2002) Comparative vertebrate lateralization. Cambridge: Cambridge UP.

Rogers LJ, Bolden SW (1991) Light-dependent development and asymmetry of visual projections. Neurosci Lett 121:63-67.

Rogers LJ, Deng C (1999) Light experience and lateralization of the two visual pathways in the chick. Behav Brain Res 98:277-287.

Rogers LJ, Rajendra S (1993) Modulation of the development of lightinitiated asymmetry in chick thalamofugal projections by oestradiol. Exp Brain Res 93:89-94.

Rogers LJ, Sink HS (1988) Transient asymmetry in the projections of the rostral thalamus to the visual hyperstriatum of the chicken, and reversal of its direction by light exposure. Exp Brain Res 70:378-384.

Rogers LJ, Workman L (1989) Light exposure during incubation affects competitive behaviour in domestic chicks. App Anim Behav Sci 23:187-198.

Rogers LJ, Zucca P, Vallortigara G (2004) Advantage of having a lateralized brain. Proc R Soc Lond B Biol Sci 271:S420-S422.

Sampath K, Rubinstein AL, Cheng AMS, Liang JO, Fekany K, Solnica-Krezel L, Korzh V, Halpern ME, Wright CVE (1998) Induction of the zebrafish ventral brain and floor plate requires Cyclops/Nodal signalling. Nature 395:185-189.

Schier AF (2003) Nodal signaling in vertebrate development. Annu Rev Cell Dev Biol 19:589-621.

Schwabl H (1999) Developmental changes and among-sibling variation of corticosterone levels in an altricial avian species. Gen Comp Endocrinol 116:403-408. 
Schwarz IM, Rogers LJ (1992) Testosterone: a role in development of brain asymmetry in the chick. Neurosci Lett 146:167-170.

Skiba M, Diekamp B, Güntürkün O (2002) Embryonic light stimulation induces different asymmetries in visuoperceptual and visuomotor pathways of pigeons. Behav Brain Res 134:149-156.

Sovrano VA, Dadda M, Bisazza A (2005) Lateralized fish perform better than nonlateralized fish in spatial reorientation tasks. Behav Brain Res 163:122-127.

Sun T, Patoine C, Abu-Khalil A, Visvader J, Sum E, Cherry TJ, Orkin SH, Geschwind DH, Walsh CA (2005) Early asymmetry of gene transcription in embryonic human left and right cerebral cortex. Science 308:1794-1798.

Sutherland RJ (1982) The dorsal diencephalic conduction system: a review of the anatomy and functions of the habenular complex. Neurosci Biobehav Rev 6:1-13.

Vallortigara G, Rogers LJ (2005) Survival with an asymmetrical brain: advantages and disadvantages of cerebral lateralization. Behav Brain Sci, in press.
Vauclair J, Yamazaki Y, Güntürkün O (2005a) The study of hemispheric specialization for categorical and coordinate spatial relations in animals. Neuropsychologia, in press.

Vauclair J, Mequerditchian A, Hopkins WD (2005b) Hand preferences for unimanual and coordinated bimanual tasks in baboons (Papio anubis). Brain Res Cogn Brain Res, 25:210-216.

von Fersen L, Güntürkün O (1990) Visual memory lateralization in pigeons. Neuropsychologia 28:1-7.

Ward J, Milliken G, Stafford D (1993) Patterns of lateralized behavior in prosimians. In: Primate laterality: current behavioral evidence of primate asymmetries (Ward J, Hopkins W, eds), pp 43-71. New York: Springer.

Ward JP, Hopkins WD (1993) Primate laterality: current behavioral evidence of primate asymmetries. New York: Springer.

Yeni-Komshian GH, Benson DA (1976) Anatomical study of cerebral asymmetry in the temporal lobe of humans, chimpanzees, and rhesus monkeys. Science 192:387-389. 